\title{
Cambios Sintomáticos en Policías con Estrés Postraumático y Psicoterapia de Grupo
}

\section{Symptomatic Changes in Police Officers with Post-Traumatic Stress and Group Psychotherapy}

\author{
Álvaro R. Vallejo \\ Pontificia Universidad Javeriana, Cali, Colombia.
}

(Rec: 2 de junio de 2010; Acep: 25 de noviembre de 2010)

\begin{abstract}
Resumen
El artículo muestra los cambios sintomáticos que tuvieron Policías colombianos ( 7 hombres y una mujer con edades entre los 18 y 45) que presentaban estrés postraumático después de un proceso de intervención psicoterapéutica grupal de orientación psicoanalítica. El proceso psicoterapéutico duró 3 meses con una frecuencia de 2 sesiones por semana y una duración de 1.30 hora por sesión. Los cambios sintomáticos fueron evaluados a través de "Escala administrada por el clínico" (Clinician-Administered PTSD Scale, CAPS; Blake, Weathers, Nagy, Kaloupek, Charney y Keane, 1995) y de entrevistas clínicas individuales. Se encontró que en los policías algunos síntomas asociados a los Criterios B, C, D, F, de la escala, se disminuyeron, otros se incrementaron, y en otros no hubo modificaciones.
\end{abstract}

Palabras clave: Psicoterapia de grupo, estrés postraumático, trauma, psicoanálisis, policías

\begin{abstract}
The article shows the changes that were symptomatic among Colombian Police officers ( 7 men and one woman aged between 18 and 45) who had post-traumatic stress after an intervention process psychoanalytically oriented group psychotherapy. The psychotherapeutic process lasted 3 months with a frequency of 2 sessions per week and a duration of 1.30 hours per session. Symptomatic changes were assessed by Scale administered by the clinician (Clinician-Administered PTSD Scale, CAPS, Blake, Weathers, Nagy, Kaloupek, Charney and Keane, 1995) and individual clinical interviews. We found that in Police officers, some symptoms associated with Criterion B, C, D, and of the F scale, decreased, in others increased, and there were no other changes.
\end{abstract}

Key words: Group's psychotherapy, stress posttraumatic, trauma, psychoanalysis, police officers.

Este artículo es producto de la investigación piloto denominada: "Efectos de la psicoterapia de grupo en policías con estrés postraumático producto de confrontaciones con la guerrilla o grupos armados al margen de la ley" realizada durante el período 2008-2009. Investigación financiada por la Pontificia Universidad Javeriana, Cali.

Correspondencia: Álvaro Roberto Vallejo Samudio, Departamento de Ciencias Sociales, Carrera de Psicología de la Universidad Javeriana, Cali, Colombia, Calle 18 N 118-250, Av. Cañasgordas. Edificio el Samán, oficina 356. E-mail: arvallejo@javerianacali.edu.co 


\section{Introducción}

El conflicto armado y la violencia que vive Colombia desde hace más de 50 años, ha tenido y tiene graves consecuencias en lo político, en lo económico y en lo social. Según De la Hoz \& Vélez (2008), del Instituto Nacional de Medicina Legal y Ciencias Forenses [IMLCF], la violencia (Homicidio) en Colombia en el 2008, se cobró la vida de 15. 251 personas. La Oficina del Alto Comisionado para los Refugiados [ACNUR] señala que en Colombia, en el año 2008, existían tres millones de personas que han tenido que huir de sus sitios de origen y se encuentran en condiciones de desplazamiento (Cadena Radial Colombiana [CARACOL], 2008).

La violencia en Colombia deja graves secuelas a corto, a mediano y a largo plazo en la salud mental, en la identidad y en la autoestima de los colombianos; incrementando los riegos de Trauma psicológico y de Estrés Postraumático (Rodríguez, De La Torre \& Miranda, 2002). Según el último Estudio Nacional de Salud Mental correspondiente al año 2003, realizado por el Ministerio de la Protección Social (2003), el índice de prevalencia del trastorno por estrés postraumático (TEPT) en la población colombiana es de $3.5 \%$, ahora, si se está directamente involucrado en el conflicto armado, este hecho se constituye como un factor de alto riesgo de sufrir de TEPT.

La investigación realizada por Pérez, Fernández, \& Rodado (2005) evidenció que los niños que viven en municipios donde han sucedido confrontaciones armadas, tienen diecinueve (19) veces mayor probabilidad de sufrir TEPT bélico que los niños de municipios no expuestos. En el análisis realizado por Londoño, Muñiz. Correa, Patiño, Jaramillo, Raigoza et al. (2005), con habitantes de Bojaya, donde se presentaron confrontaciones entre la Guerrilla y los Paramilitares, se encontró una prevalencia en la población civil de TEPT del $37 \%$.

Son inexistentes las investigaciones sobre prevalencia de TEPT en policías colombianos. Alarcón (2002) plantea que los estudios de TEPT en veteranos de guerra norteamericanos demuestran en esta población una prevalencia de síndrome entre el $12-30 \%$ y que de éstos hay una tendencia de entre $15-25 \%$ a cronificar el trastorno.

Si bien la sociedad civil en Colombia se encuentra afectada de TEPT, más lo están las personas directamente involucradas en el conflicto interno y la violencia, por una parte, los militares y policías, y por otra, los colombianos pertenecientes a los grupos armados ilegales (guerrilleros o paramilitares). La serie de situaciones complejas y difíciles que tienen que vivir diariamente los policías colombianos (tomas guerrilleras, carobombas, ataques por sorpresa, etc,) en las que se pone en riesgo su vida o la de algunos de sus compañeros, hace que muchos de ellos experimenten situaciones de altos niveles de estrés y desarrollen traumas, sufriendo graves lesiones físicas y psicológicas.

El Trastorno por estrés postraumático trae como consecuencia un gran número de dificultades en casi todas las áreas de la vida de los Policías y Militares, aunque se observan especialmente en relación a la integración familiar, social y laboral (Millán, Gujis, Diez, Storino, \& Foster (2005); Sandín (2003); Vallejo \& Zapata, 2009). Las investigaciones demuestran que tienen serias dificultades en diferentes áreas como: divorcio (70\%), arresto - problemas con la justicia (39\%), inestabilidad ocupacional (42\%), problemas maritales y parentales $(55 \%)$ y violencia $(40 \%)$ (Kulka, Schlenger, Fairbank, Jordan, Hough, Marmar \& Weiss, 1990, citados por Lubin y Read, 2000).

Existen a partir de los diferentes modelos conceptuales de la Psicología, distintas formas de intervenir terapéuticamente el TPET, las dos modalidades de intervención terapéuticas más usadas en el TPET son las individuales y la grupal.

El psicoanálisis, a partir de los trabajos de Freud y sus continuadores (Lacan, Klein, Winnicot, Kohout, Kenberg, entre otros), enfatiza la importancia del lugar de la palabra en la resignificación y comprensión del síntoma (García, 2000; Sánchez, 2003; Vallejo, 2009). La cura por la palabra o "talking cure", como denominó Ana O. a la terapia que hacía con el médico vienés, es lo que va permitir al sujeto encontrarse con sus deseos e integrar partes escindidas de sí mismo.

La intervención terapéutica grupal aparece en el contexto internacional en el siglo XX cuando, en 1905, Joseph Pratt observó que sus pacientes tuberculosos mejoraban en las sesiones de grupo cuando, además de realizar lecturas u obtener información referente a la enfermedad, se les permitía hablar acerca de los efectos y dificultades de la misma (Kaplan \& Sadock, 1996). Desde entonces, y tras un proceso de desarrollo, esta modalidad de intervención psicoterapéutica se ha constituido como un procedimiento eficaz con las que cuenta el psicólogo clínico, independientemente de su orientación teórica y de la problemática particular del analizante.

La psicoterapia de grupo es una modalidad de intervención psicoterapéutica que, aunque parte del mismo procedimiento de análisis del discurso que la psicoterapia individual, va a poner en marcha una serie de mecanismos propios (universalidad, cohesión, recapitulación del grupo familiar primario y desarrollo de técnicas de socialización, entre otros) que hacen que resulte altamente efectiva y que, ante el abordaje de determinadas problemáticas y con determinados analizantes, su utilización se asocie a un mejor pronóstico que la intervención clínica individual (Pérez-Sánchez, 1996; Orengo, y Sabbah, 2001; Yalom 2000).

La investigación hecha por Botero (2005), con soldados colombianos en proceso de rehabilitación por TEPT, ha evidenciado que la intervención terapéutica grupal es efectiva al reflejar los resultados, diferencias significativas en los síntomas y en el grado de severidad de los mismos, como consecuencia del proceso de psicoterapéutico; la intervención estuvo basada en la exposición prolongada al recuerdo traumático y en el entrenamiento en inoculación de estrés. En otra investigación realizada por Rodríguez (2006), con población desplazada (adulta y adolescente) en 
Colombia, se encontró un cambio estadísticamente significativo en esta población en relación a los síntomas asociados a TEPT después de un programa de grupo estructurado en estrategias de afrontamiento. En la investigación realizada por Campanini, Schoedl, Pupo, Costa, Krupnick, \& Mello (2010), quienes hicieron psicoterapia de grupo interpersonal, encontraron que esta modalidad de intervención terapéutica no sólo fue eficaz en la disminución de síntomas asociados a TEPT, sino también en síntomas asociados a ansiedad y depresión, consiguiéndose cambios significativos en ajuste social y calidad de vida.

Este artículo tiene como finalidad plantear los resultados de una investigación de carácter piloto, en donde se buscaba indagar si, tras la aplicación de un proceso de intervención psicoterapéutica grupal con orientación psicodinamica, se producían cambios en los síntomas clínicos en un grupo de policías colombianos diagnosticados con estrés postraumático, de acuerdo a los resultados aportados por la Escala Administrada por el Clínico (CAPS) (Clinician-Administered PTSD Scale - CAPS). Las diferencias encontradas entre la aplicación previa del CAPS (antes de iniciarse el proceso psicoterapéutico grupal) y la aplicación posterior del CAPS (después de finalizado el proceso de intervención terapéutica) muestran que la psicoterapia de grupo es beneficiosa para los policías con estrés postraumático producto de confrontaciones bélicas.

\section{Método}

\section{Diseño}

En la investigación se utilizó una metodología de carácter Cuasi-Experimental con diseño intragrupo con medidas pre y pos test.

\section{Participantes}

El grupo estuvo conformado por ocho policías, siete hombres y una mujer, con edades comprendidas entre los 18 y los 45 años, pertenecientes a la Policía Metropolitana de Cali y a la Policía Valle. Todos habían sido diagnosticados con trastorno por estrés postraumático, presentaban lesiones físicas y secuelas en recuperación, producto de confrontaciones con armas de fuego ya sea con la guerrilla o grupos armados al margen de la ley. Los sujetos fueron seleccionados de manera intencional, de una muestra inicial de 60 Policías que presentaban síntomas asociados a TEPT según los criterios diagnósticos del DSM-IV por haber estado en combate, fueron remitidos por la Clínica de la Policía. Los sujetos incluidos en la investigación finalizaron el proceso terapéutico.

\section{Instrumentos}

Para evaluar los cambios en intensidad y frecuencias en la sintomatología de los Policías con TEPT como producto de la intervención terapéutica grupal, se utilizó la Escala Administrada por el Clínico (CAPS) (Clinician-Administered PTSD Scale - CAPS) desarrollada por Blake, Weathers, Nagy, Kaloupek, Charney y Keane, (1995), la escala fue validada en Español por Bobes, Calcedo-Barba, García, Francois, RicoVillademoros, González, \& Grupo Español de Trabajo para el Estudio del Trastorno por Estrés Postraumático (2000).

Esta escala fue realizada en base a los criterios diagnósticos del DSM -IV APA (1980), con el fin de evaluar y diagnosticar los síntomas del TEPT. Consiste en una entrevista estructurada que se divide en dos componentes, el CAPS-1 en el que se consignan todas las preguntas correspondientes a la evaluación diagnóstica y el CAPS-2 dentro de las que se encuentran aquellas preguntas que evalúan la gravedad de los síntomas presentes en el sujeto. Ambos componentes, evalúan los 17 síntomas existentes en el DSM -IV y cinco características asociadas, usualmente a los Trastornos por Estrés Postraumático que corresponden: a) Culpa sobre actos cometidos u omitidos, b) Culpabilidad por haber sobrevivido, c) Reducción de la conciencia de lo que lo rodea, d) Desrealización, y e) Despersonalización.

Se realizaron grabaciones sonoras de las entrevistas clínicas individuales en las cuales se aplico el CAPS, de las sesiones del proceso psicoterapéutico y de las sesiones de seguimiento.

\section{Procedimiento}

La investigación se desarrolló entre los años 2008-2009, teniendo en cuenta las disposiciones éticas del Código Deontológico y Bioético de Psicología en Colombia, Ley 1090 de 2006. Los participantes del grupo terapéutico fueron informados previamente de los objetivos de la investigación, de sus derechos, así como también del procedimiento y el tiempo en que se llevaría a cabo.

Una vez obtenido el consentimiento informado, en una primera fase y antes de iniciar el proceso psicoterapéutico se aplicó el CAPS (Pretest), posteriormente en una segunda fase se realizó el proceso psicoterapéutico grupal, y finalmente en una tercera fase se volvió nuevamente a aplicar el CAPS (Postest).

En la investigación se trabajó con los Policías que en el CAPS arrojaban un resultado clínicamente significativo. El proceso terapéutico se desarrolló en el Sala de investigaciones aplicadas de la Universidad Javeriana Cali, entre los meses de septiembre y diciembre del 2009. En un lugar amplio y acogedor adecuado para un proceso de intervención en grupos. El proceso terapéutico tuvo una duración de tres (3) meses, durante el cual se hicieron veintidós (22) sesiones de hora y media de duración cada una (90 minutos). Al mes y medio del inicio del proceso de intervención, se realizó una sesión individual de seguimiento, con el objetivo de valorar como cada uno de los participantes había percibido y vivido el proceso.

Las sesiones se iniciaban a la hora determinada con un mínimo de tres analizantes, y no seguían un formato 
estructurado. Se dejaba que cualquiera de los analizantes empezara con el tema que considerase pertinente en el momento, facilitando en todo momento la producción de asociaciones libres tanto del sujeto hablante como de cualquier otro de los analizantes del grupo.

El proceso de intervención se llevó a cabo bajo la coordinación de un psicólogo-psicoterapeuta con experiencia en esta modalidad de intervención grupal. Adicionalmente, una profesional psicóloga desempeñaba el rol de observadora, que consistía en tomar notas escritas de todas las interacciones verbales y no verbales de los participantes (incluyendo el psicoterapeuta), y quien no podía realizar intervención alguna en el proceso.

El modelo conceptual y el procedimiento desde el que intervino el psicoterapeuta a lo largo del proceso grupal fue el propuesto por Anzieu (1998); Anzieu \& Kaës, (1989) y Kaës (2000 a, b) quienes, desde una conceptualización derivada del psicoanálisis, razonan que el proceso terapéutico grupal debe considerar al grupo como si fuera un solo sujeto. Desde esta concepción, las intervenciones del terapeuta deben apuntar al grupo como un todo y no a las personas integrantes del mismo individualmente. Para estos autores, las intervenciones del analista deben hacerse principalmente en dos momentos: a) Cuando el grupo está bajo los efectos de la resistencia, y b) Cuando se estanca la asociación libre. Esta modalidad de intervención psicoterapéutica es denominada DE GRUPO y busca utilizar al grupo como instrumento del proceso psicoterapéutico.

\section{Resultados}

Los resultados que se presentan se basan en: a) Las puntuaciones obtenidas por la muestra en la aplicación pre- post de la Escala Administrada por el Clínico (CAPS). Según Bobes y Cols (2000), en su validación de la escala al español, ésta prueba se puede calificar de dos formas; para los resultados que aquí se presentan, se tuvo en cuenta la estrategia de calificación "más restrictiva". El punto de corte recomendado por los autores para la calificación global de la prueba es de 65, índice que permite diagnosticar o no el Estrés Postraumático. b) En el análisis estadístico de la información mediante la prueba no parametrica de Wilcoxon, analizado mediante el paquete estadístico SPSS 17.0 para Windows. Se estableció un nivel de significación del 5\%.

\section{a) Puntuaciones obtenidas en el Pre-Post CAPS}

Los resultados que a continuación se muestran, corresponden a la comparación de presencia o no de la sintomatología asociada a cada uno de los seis criterios A, B, C, D, E, F y las características asociadas al trauma que tiene el CAPS para evaluar estrés postraumático. Los números que aparecen en las tablas representan al total de participantes que presentan el criterio.

Antes de iniciarse el proceso psicoterapéutico grupal se aplicó el CAPS y aparece como resultados Pre-CAPS. después de finalizado el proceso psicoterapéutico se hizo nuevamente la aplicación del CAPS y aparecen como resultados Post-CAPS.

\section{Criterio A. Exposición a un evento traumático} severo

Todos los participantes cumplieron con este criterio, ya que como se evidenció en la entrevista inicial y la aplicación del CAPS, los ocho participantes, manifestaron haber estado expuestos a un suceso traumático donde experimentaron, fueron testigos o se enfrentaron con acontecimientos que implicaron la presencia real de peligro de muerte, de lesiones graves, de amenazas a la integridad física de sí mismas o de otras y donde las respuestas de las personas incluyeron miedo intenso, indefensión o terror.

Tabla 1

Resultados comparativos del criterio B. pre-post CAPS (Actual y a lo largo de la vida) de todos los participantes.

\begin{tabular}{|c|c|c|c|c|}
\hline \multirow{2}{*}{ Síntomas reexperimentados. } & \multicolumn{2}{|c|}{ Actual } & \multicolumn{2}{|c|}{ A lo largo de la vida } \\
\hline & Pre-CAPS & Post-CAPS & Pre-CAPS & Post-CAPS \\
\hline $\begin{array}{l}\text { Recuerdos molestos e intrusivos del acontecimiento, incluyendo imágenes, } \\
\text { pensamientos o percepciones. }\end{array}$ & 7 & 6 & 8 & 5 \\
\hline Sueños angustiosos y recurrentes. & 6 & 3 & 8 & 4 \\
\hline $\begin{array}{l}\text { Actuar o sentir el acontecimiento traumático como si estuviera sucediendo } \\
\text { de nuevo. }\end{array}$ & 7 & 7 & 8 & 7 \\
\hline $\begin{array}{l}\text { Malestar psicológico intenso al verse expuesto a estímulos externos o } \\
\text { internos que simbolizan el evento traumático. }\end{array}$ & 5 & 5 & 5 & 5 \\
\hline $\begin{array}{l}\text { Reacciones fisiológicas al exponerse a estímulos internos o externos que } \\
\text { simbolizan o recuerdan un aspecto del acontecimiento traumático. }\end{array}$ & 6 & 4 & 5 & 5 \\
\hline
\end{tabular}


Criterio B. Este criterio evalúa los síntomas reexperimentados

En la tabla 1 aparecen los resultados comparativos para el criterio B, de la aplicación Pre-Post CAPS en el tiempo "Actual" y a "Lo largo de la Vida", evidenciándose en el Post-CAPS, una disminución en un $50 \%$ de la población que antes presentaban sintomatología asociada a Sueños angustiosos y recurrentes en ambos tiempos, si se compara con el Pre- CAPS. Así mismo, se evidencia que en la sintomatología asociada a Recuerdos molestos e intrusivos del acontecimiento, de siete (7) personas que lo presentaban en el Pre-CAPS, pasó a seis (6) en el Post-CAPS. En el mismo síntoma la comparación Pre y Post CAPS a lo largo de la vida, muestra que de ocho participantes que lo presentaban antes del proceso grupal, después solo lo tenían cinco.

El síntoma asociado a Reacciones fisiológicas al exponerse a estímulos internos o externos que simbolizan o recuerdan un aspecto del acontecimiento traumático, de un total de seis (6) participantes que presentaban el síntoma en el Pre-CAPS, después cuatro (4) lo presentan en el Post-CAPS, manteniéndose igual para Pre-Post CAPS a lo largo de la vida. Con relación a la sintomatología asociada a Malestar psicológico intenso al verse expuesto a estímulos externos o internos que simbolizan el evento traumático, no hubo ninguna modificación entre el Pre-Post CAPS actual y a lo largo de la vida, estando presente en cinco (5) de los ocho (8) participantes.

Con relación al síntoma Actuar o sentir el acontecimiento traumático como si estuviera sucediendo de nuevo, en relación al pre-post CAPS actual no hubo ninguna modificación, presentándolo cinco (5) de los ocho (8) participantes, en esta misma escala para el tiempo referido a lo largo de la vida se pasó de ocho (8) personas que lo presentaban a presentarlo siete (7).

Criterio C. Este criterio evalúa los síntomas de evitación y paralización.

En la tabla 2 aparecen los resultados comparativos del criterio C, entre el Pre y Post CAPS, en el tiempo Actual y a lo Largo de la vida, se evidencia que hubo una disminución en los síntomas asociados a Esfuerzos por evitar pensamientos, sentimientos o conversaciones relativos al trauma, ya que de seis personas (6) que lo presentaban, pasaron a presentarlos cuatro (4) en ambos tiempos. En la sintomatología asociada a Afecto restringido como producto del trauma, se hizo evidente la disminución del síntoma en el Post-CAPS, para el tiempo Actual, ya que antes lo presentaban cinco (5) participantes y después del proceso de intervención lo presentaron (3). Mientras que éste mismo síntoma en el Post- CAPS a lo largo de la vida pasaron de tenerlo seis (6) participantes a tenerlo cuatro (4).

Tabla 2

Resultados comparativos del criterio C. pre-post CAPS (actual y a lo largo de la vida) de todos los participantes.

\begin{tabular}{lccc}
\hline Síntomas de evitación y paralización. & \multicolumn{2}{c}{$\begin{array}{c}\text { Actual } \\
\text { A lo largo de la vida }\end{array}$} & $\begin{array}{c}\text { Post-CAPS } \\
\text { Pre-CAPS }\end{array}$ \\
\hline $\begin{array}{l}\text { Esfuerzos por evitar pensamientos, sentimientos o conversaciones relativos } \\
\text { al trauma. }\end{array}$ & 6 & 4 & 6 \\
$\begin{array}{l}\text { Esfuerzos por evitar actividades, lugares o personas que producen recuerdos } \\
\text { del trauma. }\end{array}$ & 4 & 5 & 5 \\
$\begin{array}{l}\text { Incapacidad para recordar aspectos importantes del trauma. } \\
\text { Pisminución del interés o de la participación en actividades. }\end{array}$ & 0 & 1 & 0 \\
Sensación de desapego o alejamiento. & 5 & 5 & 6 \\
Afecto restringido. & 6 & 6 & 6 \\
Sensación de un porvenir acortado. & 5 & 3 & 7 \\
\hline
\end{tabular}

El síntoma asociado a Sensación de un porvenir acortado, como efecto del trauma, en el Pre-Post CAPS actual; existió una disminución, ya que de cinco participantes (5) que lo tenían, se pasó a tres (3). En relación al Pre-Post CAPS a lo largo de la vida, de seis (6) participantes que presentaban el síntoma, se pasó a cinco (5) que lo presentan. Los resultados comparativos del criterio C, en las aplicaciones Pre-Post del CAPS, Actual y a lo Largo de a la vida, para los síntomas: (a) Sensación de desapego o alejamiento de los demás y (b) Disminución del interés o de la participación en actividades sociales, demuestran que no hubo modificación en el número de personas que lo presentaban.

Con relación a la sintomatología asociada a Esfuerzos por evitar actividades, lugares o personas que producen recuerdos del trauma, entre el Pre-Post CAPS Actual hubo un incremento, ya que antes el síntoma lo presentaban cuatro (4) participantes y después lo presentaron cinco (5). Con 
relación a éste mismo síntoma en la comparación Pre- Post CAPS a lo Largo de la vida no hubo modificación alguna, ya que lo continuaban presentando los mismos cinco (5) participantes.

Finalmente, en los síntomas asociados a la Incapacidad para recordar aspectos importantes del trauma, solo hubo una variación, puesto que una persona presenta este síntoma en la aplicación Post-CAPS en el tiempo actual. No presentándose ninguna modificación en este síntoma en el Pre-Post CAPS a lo largo de la vida.

Criterio D. Éste criterio evalúa síntomas relacionados con la Hipervigilancia

La tabla 3 evidencia que en el síntoma asociado a Sobresalto ante estímulos normales, hubo una disminución en el tiempo Actual en los participantes que presentaban el síntoma, ya que de siete (7) participantes en el Pre-CAPS se pasó a cinco (5) en el Post-CAPS, sin embargo en el tiempo denominado a lo Largo de la vida hubo un incremento en los participantes que presentaban el síntoma, ya que de cinco (5) que lo presentaban en Pre-CAPS se pasó a siete (7) en el PostCAPS. La sintomatología asociada a Irritabilidad o accesos de cólera, tuvo una disminución en el Pre-Post CAPS Actual, ya que antes lo presentaban seis (6) personas y después lo presentaron (5); en el tiempo a lo largo de la vida se pasó de (8) personas que lo presentaban, a seis (6) en el Post-CAPS.

Con relación a la Dificultad para concentrarse, se mantiene en el Pre-Post CAPS Actual, la presencia del síntoma en cinco (5) participantes. A lo Largo de la vida disminuye en un $50 \%$ las personas que lo presentan, pasando a presentar el síntomas cuatro (4) participantes en el Post-CAPS, en el síntoma Dificultad para conciliar o mantener el sueño en la aplicación Pre-Post CAPS en el tiempo Actual, se evidencia un aumento de una (1) persona que presenta el síntoma, ya que en el Pre-CAPS lo presentaban cinco (5) y en el PostCAPS lo presentan seis (6). En cuanto a este mismo síntoma a lo Largo de la vida, se presenta una disminución en tres (3) participantes, ya que de ocho (8) personas que presentaban los síntomas se paso a cinco (5) en el Post-CAPS.
Finalmente, el síntoma de Hipervigilancia en el Pre-Post CAPS actual evidencia el aumento de la sintomatología en una (1) persona, ya que se pasó de siete (7) participantes en el Pre-CAPS, a ocho (8) en el Post-CAPS. A lo Largo de la vida, de cinco (5) personas que presentaban el síntoma en el Pre-CAPS, se pasó a ocho (8) en el Post-CAPS.

Criterio E. Los síntomas de los B, C y D se prolongan más de un mes.

Éste criterio, corresponde a la prolongación por más de un mes de los síntomas de los criterios B, C y D antes mencionados, según lo afirmado por los participantes, todos presentaron los síntomas en un periodo menor a seis meses después de ocurrido el suceso traumático y que la duración de la molestia se ha mantenido por varios meses y años. En estado crónico (presencia de los síntomas mayor o igual de 3 meses) presentan este criterio, siete de los ocho participantes, correspondiente al $87.5 \%$ de la muestra; y uno de ellos en estado agudo (menor a tres meses).

Criterio F. Malestar clínico significativo o deterioro social, laboral o de otras áreas de la actividad del individuo.

La tabla 4 muestra que con relación al síntoma Angustia Subjetiva en el Pre-CAPS en el tiempo Actual, de seis (6) personas que lo presentaban se pasó cinco (5) personas que lo presentaron en el Post-CAPS. En el tiempo a lo largo de la vida se pasó de presentarlo ocho (8) participantes En el Pre-CAPS a tenerlo seis (6) en el Post-CAPS. Con relación al síntoma Interferencia con el funcionamiento social en el tiempo actual, se pasó de ocho (8) personas que lo presentaban en el Pre-CAPS actual a ser presentado por cinco (5) en el Post- CAPS; en relación al tiempo a lo largo de la vida, de ocho (8) participantes que presentaban el síntoma en el Pre-CAPS se pasó a siete (7) en el Post CAPS.

Finalmente, el síntoma denominado Interferencia con el funcionamiento laboral, pasó de presentarse en ocho (8) personas en el Pre-CAPS a ser presentado por cinco (5) en el Post- CAPS actual. En relación al tiempo a lo largo de la vida, de ocho (8) que lo exteriorizaban se pasó a seis (6) Post-CAPS.

Tabla 3

Resultados comparativos del criterio D. pre-post CAPS (actual y a lo largo de la vida) de todos los participantes.

\begin{tabular}{lcccc}
\hline \multirow{2}{*}{ Síntomas de hipervigilancia. } & \multicolumn{2}{c}{ Actual } & \multicolumn{2}{c}{ A lo largo de la vida } \\
& Pre-CAPS & Post-CAPS & Pre-CAPS & Post-CAPS \\
\hline Dificultad para conciliar o mantener el sueño & 5 & 6 & 8 & 6 \\
Irritabilidad o accesos de cólera & 6 & 5 & 8 & 8 \\
Dificultad para concentrarse & 5 & 5 & 5 & 8 \\
Hipervigilancia & 7 & 5 & 5 & 7 \\
Sobresalto ante estímulos normales & 7 & 8 & 5 \\
\hline
\end{tabular}


Tabla 4

Resultados comparativos del criterio F. pre-post CAPS (actual y a lo largo de la vida) de todos los participantes.

\begin{tabular}{lccc}
\hline $\begin{array}{l}\text { Síntomas de angustia subjetiva, interferencia en el funcionamiento social } \\
\text { y laboral. }\end{array}$ & \multicolumn{2}{c}{ Actual } & \multicolumn{2}{c}{ A lo largo de la vida } \\
\hline Angustia subjetiva & 6 & 5 & 8 \\
Pre-CAPS & Post-CAPS & 5 & Post-CAPS \\
Interferencia con el funcionamiento social & 8 & 5 & 8 \\
Interferencia con el funcionamiento laboral & 8 & 5 & 8 \\
\hline
\end{tabular}

\section{Características asociadas al trauma}

La tabla 5 muestra que el síntoma asociado a culpabilidad del superviviente, de dos (2) personas que en el Pre-CAPS actual lo tenían, en el Post-CAPS ya no lo presentaban; en relación a este mismo síntoma hubo una disminución en un $50 \%$ de las personas que lo presentaban, ya que en el Pre-CAPS, a Lo largo de la vida, lo presentaban cuatro (4) y en el Post-CAPS lo tuvieron dos (2). En la tabla también se muestra que no hubo cambio alguno en el síntoma denominado Sensación de falta de realidad (Desrealización), entre el Pre-CAPS y el Post-CAPS actual y a lo largo de la vida.

Con relación al síntoma Culpabilidad sobre actos cometidos u omitidos, se encontró que en el Pre-CAPS en el tiempo Actual hubo un incremento en una persona que presentaba el síntoma, pasando de dos (2) a tres (3) personas que lo presentan en el Post-CAPS. Lo mismo ocurrió en el Pre-Post CAPS a lo largo de la vida, ya que de cuatro personas (4) se pasó a cinco (5) personas que presentan el síntoma. En cuanto al síntoma relacionado con la Reducción de conciencia de lo que lo rodea, hubo un aumento tanto en el Pre-Post CAPS actual como en el Pre-Post CAPS a lo largo de la vida. Puesto que en el primero, pasó de presentarse en dos (2) personas a manifestarse en tres (3) y en el segundo, pasó de tres (3) a siete (7) personas.

Tabla 5

Resultados comparativos de las características asociadas. pre-post CAPS (actual y a lo largo de la vida) de todos los participantes.
Finalmente, la característica asociada a la Despersonalización en el Pre-CAPS actual pasó de no presentarse en ningún participante a manifestarse en el Post-CAPS actual en dos (2) participantes. Con relación al Pre-CAPS a lo largo de la vida, pasó de presentarse en dos (2) personas a manifestarse en una (1) en el Post-CAPS.

\section{b) Análisis estadístico}

A continuación se contrastará si luego de la intervención psicoterapéutica grupal los puntajes tanto en Síntomas Reexperimentados (Criterio B del CAPS), Síntomas de Evitación y Paralización (Criterio C del CAPS), Síntomas de Hipervigilancia (Criterio D), Características Asociadas al trauma y Puntajes Global de la prueba mejoraron o no, como producto de la intervención terapéutica. Tras el tratamiento se compararon las puntuaciones entre el pretest y el postest aportadas por CAPS mediante la prueba no paramétrica de Wilcoxon. Ningún puntaje resulta estadísticamente diferente, al nivel de significación del 5\% previamente establecido, en el pretest y el postest para el tiempo "Actual" como se muestra en la tabla 6. Es decir, que al parecer la intervención no tuvo efecto alguno sobre el estrés postraumático de los Policías.

\begin{tabular}{|c|c|c|c|c|}
\hline \multirow{2}{*}{ Características Asociadas al Trauma } & \multicolumn{2}{|c|}{ Actual } & \multicolumn{2}{|c|}{ A lo largo de la vida } \\
\hline & Pre-CAPS & Post-CAPS & Pre-CAPS & Post-CAPS \\
\hline Culpabilidad sobre actos cometidos u omitidos & 2 & 3 & 4 & 5 \\
\hline Culpabilidad del superviviente & 2 & 0 & 4 & 2 \\
\hline Reducción de la conciencia de lo que le rodea & 2 & 3 & 3 & 7 \\
\hline Sensación de falta de realidad (Desrealización) & 3 & 3 & 4 & 4 \\
\hline Despersonalización & 0 & 2 & 2 & 1 \\
\hline
\end{tabular}


Tabla 6

Prueba no paramétrica de Wilcoxon para el caso "Actual"

\begin{tabular}{cccccc}
\hline $\begin{array}{c}\text { Estadístico de } \\
\text { Prueba }\end{array}$ & Síntoma Reexperimentados & $\begin{array}{c}\text { Síntoma de } \\
\text { Evitación }\end{array}$ & $\begin{array}{c}\text { Síntoma de } \\
\text { Hipervigilancia }\end{array}$ & $\begin{array}{c}\text { Características } \\
\text { Asociadas }\end{array}$ & Total \\
\hline $\mathrm{Z}$ & -1.476 & -.842 & -.954 & -.141 & -1.439 \\
p-valor &, 140 &, 400 &, 340 &, 888 &, 150 \\
\hline
\end{tabular}

Tabla 7

Prueba no paramétrica de Wilcoxon para el caso "A lo largo de la vida"

\begin{tabular}{cccccc}
\hline $\begin{array}{c}\text { Estadístico de } \\
\text { Prueba }\end{array}$ & Síntoma Reexperimentados & $\begin{array}{c}\text { Síntoma de } \\
\text { Evitación }\end{array}$ & $\begin{array}{c}\text { Síntoma de } \\
\text { Hipervigilancia }\end{array}$ & $\begin{array}{c}\text { Características } \\
\text { Asociadas }\end{array}$ & Total \\
\hline $\mathrm{Z}$ & -2.383 & -1.332 & -.141 & -.140 & -1.183 \\
p-valor &, 017 &, 183 &, 888 &, 889 &, 237 \\
\hline
\end{tabular}

En cuanto al tiempo "A lo largo de la vida", el puntaje de Síntoma Reexperimentados resulta estadísticamente diferente, al nivel de significación del 5\%, en el pretest y el postest (Wilconxon, $\mathrm{Z}=-2.383$; $\mathrm{p}=0,017$ ); sin embargo en los demás puntajes no existen diferencias significativas (tabla 7).

\section{Discusión}

Según los resultados del análisis estadístico, por una parte, se concluye que en esta investigación piloto no existieron cambios estadísticamente significativos en la sintomatología de Policías con estrés postraumático espués de un proceso psicoterapéutico grupal de tres meses de duración, si se toma como referencia las puntuaciones obtenidas en el Pre-Post CAPS, siendo la excepción el Criterio B de la escala en el tiempo a lo Largo de la vida, en donde sí habrían diferencias significativas (Wilconxon, $\mathrm{Z}=-2.383 ; \mathrm{p}=0,017)$ presumiblemente debidas al proceso psicoterapéutico.

Por otra parte, habría que matizar lo antes planteado, ya que los resultados de la investigación muestran si se tiene en cuenta las puntuaciones de los criterios del CAPS para evaluar la presencia o ausencia de síntomas en Policías con estrés postraumático, que el proceso psicoterapéutico grupal fue relativamente efectivo, ya que si bien no hubo cambios estadisticamente significativos si se dio una disminución en algunos de los síntomas que evalúa la escala.

Hubo una disminución en los siguientes síntomas asociados a los Criterio, B, C, D, F. y Características Asociadas, para ambos tiempos (Actual y a lo Largo de la vida), después del proceso de intervención terapéutico grupal de orientación psicoanalítica en: a) Sueños angustiosos y recurrentes, b) Recuerdos molestos e intrusivos del trauma incluyendo imágenes, pensamientos o percepciones, c) Esfuerzos por evitar pensamientos, sentimientos, o conversaciones sobre el suceso traumático, d) Afecto restringido, e) Sensación de un porvenir acortado, f) Irritabilidad o accesos de cólera, g) Angustia subjetiva, h) Interferencia en el funcionamiento social, I) Interferencia con el funcionamiento laboral. De las características asociadas hubo una disminucion según el CAPS en el síntoma denominado Culpabilidad del superviviente.

Además, disminuyeron los siguientes síntomas asociados a los criterios antes expuestos para el tiempo Actual: a) Reacciones fisiológicas al exponerse a estímulos internos o externos que simbolizan o recuerdan un aspecto del acontecimiento traumático, b) Sobresalto ante estímulos normales. También, hubo disminución en el tiempo a lo Largo de la vida para los siguientes síntomas: a) Actuar o sentir el acontecimiento traumático como si estuviera sucediendo de nuevo, b) Dificultad para conciliar o mantener el sueño, c) Dificultad para concentrarse.

La explicación a esa disminución sintomática puede deberse si se tiene en cuenta los relatos verbales de los Policías, a que durante el proceso psicoterapéutico, ellos desarrollaron entre sí y al interior del grupo, una confianza básica en el otro y en sí mismos que antes no existía, en la medida que pudieron significar de forma diferente el hecho traumático y percibir que no eran los únicos que habían pasado por una situación tan compleja y difícil y, que las fantasías y reproches que se hacían cada uno, también en algún momento la habían tenido y vivenciado los otros. Asímismo, al escuchar los relatos de sus compañeros se generó un ambiente de respeto, confidencialidad y tranquilidad al interior del grupo que les facilitó hablar, sin sentirse criticados o juzgados por lo que pensaban, decían, o contaban que hicieron, generándose por consiguiente una disminución de la angustia frente a sueños, recuerdos, y pensamientos de carácter intrusivo, o de conversaciones 
que les recordaban la experiencia traumática, coincidiendo los resultados encontrados en este sentido con lo planteado por Orengo, \& Sabbah (2001).

Al poder contar a pares lo que sentían y pensaban, permitió que una cantidad de afectos que antes estaban reprimidos o estancados salieran a luz y generaran, además, de una sensación de bienestar, la posibilidad de que los policías asimilaran la experiencia traumática desde una perspectiva distinta, encontrando que el futuro podía ser pensado y vivenciado de forma diferente al que hasta hace un tiempo cavilaban. En este sentido el grupo ayudó a cada uno de los Policías a que comprendieran que si bien allí compartían el dolor y las secuelas que dejo consigo el hecho traumático, era una realidad que ellos debían asumir sus vidas y las consecuencias que éste trajo. A partir del reconocimiento de su propia soledad e individualidad, ellos reflexionaron sobre cómo retomar sus vidas de una manera diferente, coincidiendo los resultados encontrados en este sentido con lo planteado por Yalom (2000).

Los resultados tan bien evidencian que hubo otros síntomas asociados a los criterios, B, C, E y características asociadas que no se modificaron y que lo presentaron el mismo número de Policías que lo presentaban antes de hacerse el proceso psicoterapéutico. Estos síntomas serían los siguientes para ambos tiempos: a) Malestar Psicológico intenso al verse expuesto a estímulos externos o internos que simbolizan el evento traumático, b) Disminución del interés o de la participación en actividades, c) Sensación de desapego o alejamiento. Así mismo, se mantuvieron sin modificación alguna en el tiempo Actual los siguientes síntomas: a) Actuar o sentir el acontecimiento traumático como si estuviera sucediendo de nuevo, b) Dificultad para concentrarse. De las Características asociadas al trauma, después del proceso de intervención psicoterapéutico grupal tampoco hubo modificación en el síntoma de Sensación de falta de realidad (Desrealización).

La explicación a la no modificación de los síntomas antes enunciados podría deberse a que los efectos del trauma hacían que pese al proceso psicoterapéutico grupal, los Policías siguieran estando muy sensibles a cualquier evento externo o interno que les evocara el trauma, lo que lleva a considerar que si bien ellos no se resistían como antes de la intervención terapéutica para pensar, recordar o hablar del suceso traumático, y para expresar en sus núcleos familiares más cercanos el afecto, ello no implicaba que no se sintieran vulnerables a eventos externos o internos que les recordara el trauma, por ello, buscaban alejarse de las personas, lugares o actividades que los llevaban a revivir o recordar el trauma, generándose una inhibición para disfrutar de actividades que antes les producían satisfacción y haciendo que se aislaran de los demás. La persistencia de estos síntomas demuestra que no se dieron cambios estructurales a raíz del proceso psicoterapéutico.
Debe considerarse como una medida defensiva en cierta medida adaptativa de los Policías, el no que querer frecuentar los lugares en donde estuvo en peligro la vida; no así cuando pensaban que si se alejaban de las personas con las que compartieron o fueron testigos del trauma, éste iba a mejorar o no iba a causarles dolor y tristeza. La presencia de hipervigilancia y sobresalto ante estímulos normales, por una parte, denota necesariamente que no sabían diferenciar entre algo que ya sucedió y algo que está aconteciendo ahora. Como si una parte de ellos estuviera siempre temerosa de que "algo malo" podía sucederles, "viviendo" en el tiempo en que sucedió el evento traumático. Por otra parte, la razón del incremento de la sintomatología asociada a hipervigilancia en los Policías, puede explicarse si se tiene en cuenta el discurso verbal de los mismos, que en un país como Colombia donde el conflicto armado y los enfrentamientos de la Policía con las bandas al margen de la ley es un hecho real y de todos los días, el presentar estos síntomas tendría paradójicamente una función de índole adaptativa ya que ellos lograron dimensionaron gracias a la intervención terapéutica, con mayor agudeza las serias implicaciones que tuvo para su salud, su vida afectiva, social y laboral, el trauma; en razón de ello es comprensible que estén más alertas y a la defensiva para "evitar" que un suceso como los que vivieron pueda volverles a ocurrir y, de allí un aumento en la hipervigilancia

El aumento en los síntomas de las Características asociadas al trauma, por una parte, tiene que leerse a la luz de una mayor conciencia que los Policías podrían haber desarrollado, durante la intervención terapéutica, de lo que hicieron o dejaron de hacer, para que el evento traumático sucediera, ya sea en la realidad o en su fantasía; por otra parte, el aumento en la sintomatología asociada a la "culpa" podría tener su explicación en el hecho de que los Policías antes del proceso terapéutico no habían hecho conscientes afectos y representaciones asociados a ésta, y a medida que fue avanzando la psicoterapia lo consiguieron, ya que se permitieron verbalizar situaciones complejas y actuaciones fuera de la ley.

Cuando se presentan resultados de procesos de intervención psicoterapéutica, es importante tener en cuenta que las investigaciones dedicadas a tratar de evaluar los efectos psicoterapéuticos de determinadas modalidades de intervención (grupos, individual, parejas, etc) en cualquier sintomatología (trauma, depresión, etc) son complejas y de resultados en gran medida inciertos y discutibles, ya que involucran muchos factores, que no necesariamente están bajo el control del terapeuta. Factores que Botella (2000) denomina inespecíficos y que podrían ser los cruciales a la hora de explicar los cambios existentes o no después de un proceso terapéutico.

Los resultados de esta investigación de carácter piloto en mayor o menor medida podrían haberse visto afectados debido a tres variables, a saber: 
a. El tiempo de duración del proceso

b. La cultura Policial donde se busca obtener ventajas de la "enfermedad".

c. El tamaño de la muestra y la falta de la existencia de un grupo control.

Con relación al tiempo de duración, creemos que éste influyó en los resultados encontrados, en la medida en que no es posible esperar que en veintidós (22) sesiones de hora y media de duración, que duró el proceso psicoterapéutico grupal, se puedan realizar cambios sintomáticos significativos en un trastorno de las características del estrés postraumático; mas teniendo en cuenta la gravedad de los eventos traumáticos que vivieron los Policías colombianos que participaron en la Investigación. En algunos de participantes, el evento traumático no solo expuso su vida en peligro, sino que le quedaron secuelas físicas para toda la vida, como pérdida de la mitad de la audición, o incapacidad para caminar normalmente, entre otras, etc. Otros vieron cómo morían calcinados o desmembrados sus compañeros; otra participante, además, de recibir dos balas en el cuello que casi la incapacitan para mover su cabeza, fue testigo de cómo asesinaban a su esposo delante de sus dos hijos.

La cultura existente en los Policías de buscar obtener ganancias (indemnizaciones, pensiones, cambios de puestos y lugares de trabajo) a través de sus dificultades de salud, podría haber incidido en las puntuaciones altas que se obtuvieron en el Post-CAPS, ya que ellos sabían que la División de Sanidad de la Policía otorgaba incapacidades y otras prebendas a aquellos integrantes de la policía que certificaran la gravedad de sus dificultades médicas y psicológicas. Es de puntualizar que desde el principio de la investigación y en el consentimiento informado que ellos firmaron para participar en la investigación, se hizo total claridad de que en ningún caso los investigadores darían certificados, constancias o informes que ellos puedan utilizar para buscar cualquier tipo de remuneración laboral o económica; aun así, dicha cultura estuvo muy presente en lo policías que participaron en la investigación, ya que algunos de ellos solicitaban explícitamente se les dieran constancias de diferente índole relacionadas con su trauma y el proceso terapéutico que recibían, constituyéndose esta "Cultura" en una variable extraña que puede haber afectado la validez interna de la investigación.

Los resultados de la investigación no pueden ser concluyentes en la medida en que la muestra es pequeña, ocho (8) policías, lo que no permite que se pueda hacer generalizaciones válidas a la población en general de los policías colombianos.

Para futuras investigaciones, si se desea obtener mayor validez externa e interna y confiabilidad en los resultados, se hace necesario, además, de una mayor muestra, una duración más extensa en el tiempo del proceso psicoterapéutico, y establecer un grupo control que permita verificar y contra- tar, si los resultados muestran diferencias significativas entre aquellos que reciben el tratamiento y aquellos que no.

\section{Referencias}

Alarcón, R. (2002). Trastorno por estrés postraumático: Estudios en veteranos de guerra norteamericanos y su relevancia para América Latina. Revista Chilena de Neuropsiquiatría, 40. 35-47.

Asociación Psiquiátrica Americana (2002). Manual diagnóstico y estadístico de los trastornos mentales (DSM-IV) (4a. ed.). Barcelona: Elsevier/ Masson.

Anzieu, D. (1998). El grupo y el inconsciente: Lo imaginario grupal. Barcelona: Biblioteca Nueva.

Anzieu, D. \& Kaës, R. (1989). Crónica de un grupo. México, D.F.: Gedisa.

Blake, D., Weathers F., Nagy L., Kaloupek, D., Charney, D., \& Keane, T. (1995). The development of a clinician-administered PTSD scale. Journal of Traumatic Stress, 8, 75-90.

Bobes, J., Calcedo-Barba, A., García, M., Francois, M., Rico-Villademoros, F., González, M., \& Grupo Español de Trabajo para el Estudio del Trastorno por Estrés Postraumático. (2000). Evaluación de las propiedades psicométricas de la versión española de cinco cuestionarios para la evaluación del trastorno de estrés postraumático. Actas Española de Psiquiatría, 28, 207-218.

Botella, J. (2000). El papel de los estudios de resultados de las terapias psicológicas. Psychotema, 12, 176-179.

Botero, C. (2005). Efectividad de una intervención cognitivo-conductual para el trastorno por estrés postraumático en excombatientes colombianos. Universitas Psychologica, 4, 205-220.

Campanini, R., Schoedl, A., Pupo, M., Costa, A., Krupnick, J., \& Mello, M. (2010). Eficacia del formato de terapia de grupo interpersonal adaptado al trastorno de estrés post-traumático: Un ensayo adicional abierto. Depression and Anxiety, 27, 72-77. Recuperado en Febrero 24, 2010 de http://www3.interscience.wiley.com/cgi-bin/fulltext/123209217/ HTMLSTART

CARACOL, (2008, Junio 17). ACNUR, Colombia dice que el país no es el primero del mundo en desplazamiento (Actualidad). Cadena Radial Colombiana. Recuperado en Febrero 23, 2010 de http://www.caracol. com.co/nota. aspx ?id=616414

De la Hoz, G. \& Vélez, M. (2008). Homicidios en Colombia. Recuperado Febrero 23, 2010 de http://www.medicinalegal.gov.co/index. php?option=com_wrapper\&view $=$ wrapper\&Itemid $=60$

García, A. (2000).Teoría psicoanalítica. Madrid: Biblioteca Nueva.

Kaës, R. (2000a). Las teorías Psicoanalíticas del grupo. Buenos Aires: Amorrortu Editores.

Kaës, R. (2000b). El aparato psíquico grupal. Buenos Aires: Gedisa.

Kaplan, H. \& Sadock, B. (1996). Terapia de grupo. Madrid: Medica Panamericana.

Kulka, R., Schlenger, W., Fairbank, J., Jordan, B., Hough, R., Marmar, C. \& Weiss, D. (1990). Trauma and the Vietnam War generation. En H. Lubin \& D. Read, (2000) (Edts.), Interactive psychoeducational group therapy in the treatment of authority problems in combat-related posttraumatic stress disorder. International Journal of Group Psychotherapy, 50, 277-96. Recuperado en Mayo 13, 2008, de http://proquest. umi. $\mathrm{com} /$ pqdweb index $=1 \&$ did $=55528435 \&$ SrchMode $=2 \&$ sid $=1 \& \mathrm{~F}$ $\mathrm{mt}=6 \&$ VInst $=$ PROD $\&$ VType $=P Q D \& R Q T=309 \&$ VName $=P Q D \& T S=$ $1221158332 \&$ clientId $=53504$.

Londoño N., Muñiz O., Correa J., Patiño C., Jaramillo, G., Raigoza J. et al., (2005). Salud mental en víctimas de la violencia armada en Bojayá (Chocó, Colombia). Revista Colombiana de Psiquiatría, 34, 493-505.

Millán, L., Gujis, A., Diez, G., Storino, R. \& Foster, J. (2005). Esos Loquitos de Guerra. Buenos Aires: Universidad Nacional de Lomas de Zamora.

Ministerio de la Protección Social. (2003). Estudio nacional de salud mental. Recuperado en Noviembre 10, 2009 de http://www.minpro- 
teccionsocial.gov.co/VBeContent/library/documents/DocNewsNo14822DocumentNo1466.PDF

Orengo, F., \& Sabbah, D. (2001). Trastorno de estrés postraumático: Entrevista a Chaim F. Shatan. Aperturas Psicoanalíticas. Recuperado en Noviembre 18, 2006 de http://www.aperturas.org/articulos. php?id=0000184\&a=Trastorno-de-estres-postraumatico-entrevistacon-Chaim-F-Shatan.

Pérez, I., Fernández, P., \& Rodado, S. (2005). Prevalencia del trastorno por estrés postraumático por la guerra, en niños de Cundinamarca, Colombia. Revista de Salud Pública, 7, 268-280.

Pérez-Sánchez, A. (1996). Prácticas psicoterapéuticas: Psicoanálisis aplicado a la asistencia pública. Barcelona: Paidós.

Rodríguez, J., De La Torre, A., \& Miranda, C. (2002). La Salud Mental en Situaciones de Conflicto Armado. Biomédica, 22, 337-346.
Rodríguez, M. (2006). Eficacia de un programa de grupo estructurado en estrategias de afrontamiento para DSPT en adultos y adolescentes en situación de desplazamiento. Universitas Psychologica, 5, 259-274.

Sánchez, T. (2003). Psicoanálisis y psicología convergencia o confrontación. Madrid: Biblioteca Nueva.

Sandín, B. (2003). Estrés y factores sociales. Revista Internacional de Psicología Clínica y de la Salud, 3, 141-157

Vallejo, Á. (2009). El trabajo de interpretación (deutungsarbeit) como un proceso racional-consciente en la obra de Freud. Acta Colombiana de Psicología, 12, 41-52.

Vallejo, Á., \& Terranova, L. (2009). Estrés postraumático y psicoterapia de grupo en militares. Terapia Psicológica, 1, 103-112.

Yalom, I. (2000). Psicoterapia existencial y terapia de grupo. Barcelona: Paidós. 